\title{
Molecular modeling, synthesis, and evaluation of 1-(4-methoxybenzofuran-5-yl)-3-phenylpropane-1, 3-dione for its anxiolytic potentiality
}

\author{
P Ayash Kumar ${ }^{1}$, Sanjita Das ${ }^{2 *}$, Sradhanjali Mohapatra ${ }^{3}$, Saumya Priya Basu² ${ }^{2}$ Kalicharan Sharma ${ }^{3}$, Monika Sharma ${ }^{2}$, Aniruddh \\ Pratap Singh ${ }^{4}$ \\ ${ }^{1}$ Research and Development Centre, Sun Pharma, Gurugram, India. \\ ${ }^{2}$ Noida Institute of Engineering and Technology (Pharmacy Institute), Greater Noida, India. \\ ${ }^{3}$ School of Pharmaceutical Education and Research, Jamia Hamdard, New Delhi, India. \\ ${ }^{4}$ Anand College of Pharmacy, Agra, India.
}

\begin{tabular}{l}
\hline ARTICLE INFO \\
\hline Received on: 29/03/2020 \\
Accepted on: 08/06/2020 \\
Available online: 05/08/2020 \\
\hline Key words: \\
MPD, gross behavior, \\
anxiolytic, molecular \\
modeling, GABA $_{\mathrm{A}}$ receptor.
\end{tabular}

\section{INTRODUCTION}

1-(4-methoxybenzofuran-5-yl)-3-phenylpropane-1,3dione has traditionally been acquired from natural sources mainly from the extracts of fruits of Pongamia pinnata and Pongamia glabra. 1-(4-methoxybenzofuran-5-yl)-3-phenylpropane-1,3-dione is known to have been reported for use in bronchitis, whooping cough, and rheumatic joints, to some extent as antidiabetic (Kirtikar and Basu, 1995). 1-(4-methoxybenzofuran-5-yl)-3-phenylpropane-

\section{${ }^{*}$ Corresponding Author}

Sanjita Das, Noida Institute of Engineering and Technology (Pharmacy Institute), Greater Noida, India.E-mail: sanjita8@yahoo.co.in

\begin{abstract}
The disabling mental illness anxiety is gradually affecting the modern society in any age group worldwide. The search for novel bioactive entity from herbal origin for different disorders has become the center of attraction significantly be responsible for the anxiolytic activity of most of the potent anxiolytic agents. All the available data of pongamol 1-(4-methoxybenzofuran-5-yl)-3-phenylpropane-1, 3-dione (MPD) were based on natural or semi-synthetic source. traditionally been acquired from natural sources mainly from the extracts of fruits of Pongamia pinnata and Pongamia glabra, where the yield value and the yield time are the main drawbacks. Keeping in view of the above aspects in the present research, it was approached to synthesize and evaluate the anxiolytic potential 1-(methoxybenzofuran-5yl)3-phenylpropane-1, 3-dione on experimental animals and docking procedure after its synthesis. The study of MPD on the gross behavior of mice showed a significant Central Nervous System (CNS) depressant effect. Furthermore, its anxiolytic activity was confirmed by observing its reduced locomotion of mice using actophotometer and elevated plus-maze apparatus. The highest docking score was observed to be -3.22 than the diazepam $(-3.21)$ against Gamma Amino Butyric Acid-A $\left(\mathrm{GABA}_{\mathrm{A}}\right.$. The present study provides a promising anxiolytic agent, MPD, which has its potency due to the $\mathrm{GABA}_{\mathrm{A}}$ receptor binding and causing the mitigation of the symptoms of anxiety.
\end{abstract}

1,3-dione (MPD) is reported to have anticonvulsant (Ramdhave et al., 2011), anti-oxidant and dyslipidemic (Bhatia et al., 2008), antihyperglycemic (Kumar et al., 2011), sedative (Rao et al., 2001), antiviral (Basu et al., 1994), antibacterial (Kumar et al., 2016), and anticancer (Anderson, 2003) properties which suggest it to be a potential moiety having multiple biological activity.

Anxiety is becoming a major health problem in the modernized world. The work pressure for the elders and the competition and academic pressure for the young ones are the main reasons for the increasing occurrence of anxiety. Anxiety can be distinguished from fear as a nonspecific state of heightened awareness and apprehension, whereas fear is directed at a specific identified threat. Anxiety is a part of 
the normal behavioral repertoire and is of value as a defense mechanism in raising awareness and modernized society, and the anxiety is becoming a major health problem. A search for a better therapeutic agent for the treatment of anxiety is going on as a major field of research. The brain circuits in the amygdala are thought to comprise the inhibitory networks of $\gamma$-aminobutyric acid-ergic Gamma-aminobutyric acid (GABAergic) interneurons, and this neurotransmitter, thus, plays a key role in the modulation of anxiety responses both in the normal and pathological states. The presence of allosteric sites on the Gamma Amino Butyric Acid-A $\left(\mathrm{GABA}_{\mathrm{A}}\right)$ receptor allows the level of inhibition of neurons in the amygdala to be regulated with exquisite precision, and these sites are the molecular targets of the principal classes of anxiolytic drugs. Changes in the levels of endogenous modulators of these allosteric sites as well as changes in the subunit composition of the $\mathrm{GABA}_{\mathrm{A}}$ receptor may represent mechanisms, whereby the level of neuronal inhibition is downregulated in pathological anxiety states. Neurosteroids are synthesized in the brain, which act as allosteric modulators of the GABA $\mathrm{A}_{\mathrm{A}}$ receptor. Since their synthesis is itself regulated by stress and anxiogenic stimuli, targeting the neurosteroid-GABA $\mathrm{A}_{\mathrm{A}}$ receptor axis represents an attractive site for the modulation of anxiety (Lydiard, 2003; Nuss and Gauthier, 2015). All the available data of MPD were based on natural or semisynthetic source. This encouraged toward going for a synthetic source MPD and industrial friendly process. MPD was using easily available source and quick, costeffective, and high-yielding process. MPD also exhibits antipsychotic activity (Kumar et al., 2019). Hence, it encouraged us to proceed on the industrial oriented synthesis of MPD and simultaneously explore for the anxiolytic activity of MPD. An effort has been taken to evaluate the antianxiety potential of MPD using animal models and docking studies.

\section{MATERIALS AND METHODOLOGY}

\section{Synthesis of MPD}

MPD was synthetically prepared in the study in optimized laboratory condition (Kumar et al., 2016; Mohammad, 2016). The process of substituted benzofuran with an assessment of risks is designed with an intention to make it robust, productive, cost-effective, and efficient and delivers a consistent quality. The route of synthesis is designed in the laboratory scale to meet the process requirements. The process flow chart of the final method has been illustrated as follows for the synthesis of MPD (Fig. 1). The compound was purified in column chromatography using silica gel as stationary phase and suitable solvent system. The compound has good solubility in alcohols, dimethylformamide, dimethyl sulfoxide, and toluene dichloromethane. The compound was dried in rotavapor under a vacuum. Chromatographic purity (95.66\%) of the compound was ensured in High Performance Liquid Chromatography (HPLC). It is an off white powder with a melting point of $125^{\circ} \mathrm{C}$. All the products synthesized in the route were characterized through Fourier Transform Infrared (FTIR), Nuclear Magnetic Resonance (NMR), and Mass (Kumar et al., 2016). The products were easily interpreted, and they showed stretching and bending of the functional groups as in FTIR and peaks as in NMR and Mass spectrophotometer. The reaction $\%$ yield of the process is $57 \%$. The synthesized compound was subjected to the screening of anxiolytic activity.

\section{Software used}

Schrödinger-10.6 software Protein Preparation Wizard; LLC, New York, NY, Glide Schrödinger, LLC; New York, NY.

\section{Experimental animals}

The pharmacological experiments were performed on the laboratory animals such as male Swiss albino mice weighing 20-30 g as per the norms of the Institutional Ethics Committee. The animals were kept in clean polypropylene cages in a maintained environment of temperature $\left(22 \pm 5^{\circ} \mathrm{C}\right)$, humidity $(45 \%-55 \%)$, and 12-hours light-dark cycle with proper access to the standard diet of mice pellet and water. Post acclimatization, they were separated into five groups, each group with six mice. The experimental protocol has been approved by the institutional animal ethics committee (NIET/IAEC/2011/01/11; CPCSEA Reg. No.1845/PO/Re/S/16/CPCSEA).

\section{Effect of MPD on gross behavior of mice}

Swiss albino mice were kept under observation of their behavior for 15 days. The different behavioral changes such as righting reflex, pinna reflex, corneal reflex, muscle tone, motor activity and traction test, stereotyped behavior, awareness. The individual behavior was monitored at different time intervals to record an appropriate change. These were monitored through laboratory equipment following conventional methodology (Kumar et al., 2019).

\section{Effect of MPD on locomotor activity of mice}

In this experiment, mice weighing about 20-30 g were considered. Six animals were placed in a group, and five groups were made. Actophotometer was turned on and verified for its working condition of its photocells. Each animal was placed in the instrument for 10 minutes. The basal activity score was noted.

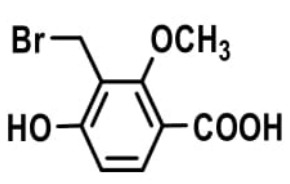

3-(bromomethyl)-4-hydroxy-2methoxybenzoic acid

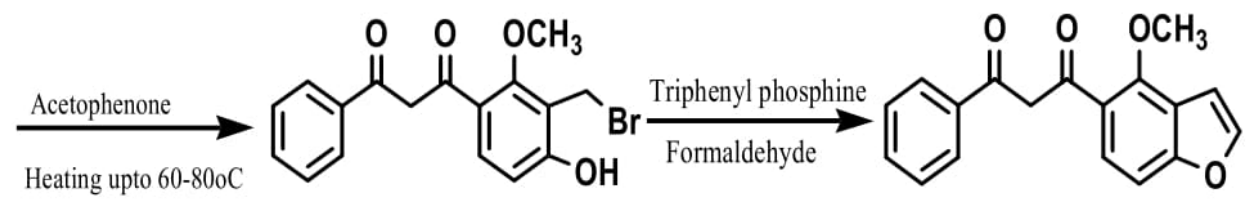

1-(4-methoxybenzofuran-5-yl)-3-phenylpropane-1,3-dione (MPD)

Figure 1. Process map for synthesis of MPD. 
Group 1 was considered with Dimethyl sulfoxide (DMSO) (1\% solution) as control, group 2 administered with diazepam $2 \mathrm{mg} /$ $\mathrm{kg}$ ) as standard, and groups 3, 4, and 5 were administered with MPD orally as test drug with a dose of 25,50 , and $100 \mathrm{mg} / \mathrm{kg}$, respectively. After 30 minutes or administration, each animal was retested for its basal activity scores (Ghosh, 1984; Turner, 1965).

\section{Anxiolytic activity of MPD using elevated plus maze}

The elevated plus maze consists of two open arms and two enclosed arms placed opposite to each other. These arms have a dimension of $50 \times 10 \times 40 \mathrm{~cm}$ with an open roof. The maze was placed at a height of $50 \mathrm{~cm}$. Six mice were taken in a group, and five groups were made. Group 1 was considered as control and administered with DMSO ( $1 \%$ solution), group 2 administered with diazepam $(2 \mathrm{mg} / \mathrm{kg})$ as standard drug, and groups 3,4 , and 5 were administered with MPD as test drug with a dose of 25 , 50 , and $100 \mathrm{mg} / \mathrm{kg}$, respectively. After administration, the rat was placed in the center of maze facing the enclosed arms. The animals were observed for time spent in open arm and a number of entries to open arms and closed arms for 5 minutes on the elevated plus maze (Ghosh, 1984; Turner, 1965).

\section{Docking studies of MPD for its anxiolytic activity}

For a better understanding of binding mode of MPD at the molecular level, molecular docking simulations of MPD were carried out at the $\mathrm{GABA}_{\mathrm{A}}$ protein receptor catalytic ligand-binding site (PDBID: 4COF). The docking of MPD was performed using Maestro, version 9.4 implemented from the Schrodinger software suite. The ligands were sketched in 3D format using a build panel and were prepared for docking using LigPrep application. The protein for docking study was taken from Protein Data Bank (PDB ID: 4COF) and prepared by removing a solvent, adding hydrogen, and further minimizing the presence of bound ligand (BEN) using protein preparation wizard. Grids for molecular docking were generated with a bound co-crystallized ligand. For the validation of docking parameters, the co-crystal ligand (BEN) was re-docked at the catalytic site of protein, and the root-mean-square deviation (RMSD) between co-crystal and re-docked pose was found to be 0.49 A. MPD was docked using Glide extraprecision mode, with up to three poses saved per molecule. The docking studies were performed using a Glide module of the Schrodinger-10.6 software on the $\mathrm{GABA}_{\mathrm{A}}$ receptor (PDB id: 6CM4). The calculations of docking were carried out using the constraint grid protocol through grid points. The binding site was defined with the confinement of the translations of the mass at the center of co-crystallized ligand. All the default parameters were used, which were considered during grid generation. Glide constraints are receptor protein, and ligand interactions favor binding with receptor. This is dependent on the structural or biochemical data of the protein setting constraints that enable Glide to screen out ligands, conformations that do not meet the examining criteria before docking (Katsila et al., 2016).

The work for the present study was performed on the Windows-7 operating system installed on Hewlett-Packard (HP) compact desktop. The virtual screening was performed on Schrodinger molecular modeling suite, and a validation (Kumar et al., 2016) was done on GOLD 5.1 software (Friesner et al., 2006; Jones et al., 1997).

\section{Database preparation}

The database is prepared for virtual screening using databases which includes from the National Cancer Institute database, Molecular Diversity Preservation International database, Maybridge Hit Finder database, Drug Bank database, and Knowledge Base database. The National Cancer Institute database and Drug Bank database release nearly 265,242 compounds that were used for virtual screening. The databases were downloaded from their respective websites, whereas knowledge-based database was developed in house through thorough sketching of compounds those reported already with little alterations using maestro graphical user interface of Schrödinger. Hence, the ligands from downloaded databases are stored and imported to Schrödinger project table as 3D format (Viswanadhan et al., 1989).

\section{Screening of databases}

Molecular modeling for docking in Schrödinger includes three steps that include Glide high-throughput virtual screening docking, Glide standard precision docking, and Glide extra precision docking. Glide high-throughput virtual screening docking is intended for the rapid screening based on similarity in terms of shape with the active site. Glide standard precision docking and Glide extra precision docking use a series of hierarchical filters to search for possible locations of the ligand in the active site of target protein. Compounds $(\sim 256,000)$ obtained after Absorption, Distribution, Metabolism, Excretion and Toxicity (ADMET) filtration were subjected to Glide high-throughput virtual screening docking, and $\sim 12,800$ hits identified were subjected to glide extra precision docking (Viswanadhan et al., 1989).

\section{RESULTS AND DISCUSSION}

\section{Effect of MPD on gross behavior of mice}

For the behavioral study, the results showed that the drug has a less depressant effect on reflexes. The drug was found to have muscle relaxant property as well as have a depressant effect on locomotor activity, which were well comparable to that of MPD and the standard drug chlorpromazine. In pharmacological screening, the animals treated with MPD were observed to have a potential inhibitory effect on catalepsy in mice suggesting its depressant effect on Central Nervous System (CNS). Although there was less effect on the reflexes, MPD was found to have a prominent inhibition in stereotaxic behavior and so causes muscle relaxation which was observed from the traction test. MPD from the pharmacological evaluation illustrates that it certainly possesses anxiolytic activity.

\section{Effect of MPD on the locomotor activity of mice}

The synthesized compound MPD was subjected to observe its effect on CNS by using actophotometer. The changes in the activity of animals were observed before and after the administration of MPD and the standard drug in actophotometer. The results of the actophotometer are shown in Table 1. The activity of the animals was found to be reduced by the administration of MPD and the standard drug diazepam. MPD showed a significant activity reduction in a dose-dependent manner which supported its CNS-depressant activity. It was observed that MPD was having 
more potential activity reduction than that of the standard drug diazepam.

\section{Anxiolytic activity of MPD using elevated plus maze}

In the present study, the elevated plus maze was used to observe the anxiolytic activity of the targeted compound MPD. Time spent on each animal in open and enclosed arms was noted. The results of the elevated plus maze are shown in Table 2. It has been observed that MPD showed a significant increase in the number of entries to open arm. The animals were found to spend more time in the open arm than the control group. It has also been observed that MPD at $100 \mathrm{mg} / \mathrm{kg}(9.25 \pm 1.89)$ found to have a well comparable anxiolytic activity than that of the standard drug diazepam $(9.25 \pm 1.89)$.

\section{Docking studies of MPD for its anxiolytic activity}

The docking studies of MPD were performed to get a better comprehension of the GABA receptor inhibitory potency at the molecular level and to shed light on the interactions in the

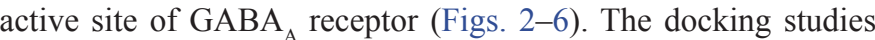
were performed using the Glide module of the Schrodinger-9.4 software on the GABA receptor (PDB id: $4 \mathrm{COF}$ ). The redocking of reference ligand (Diazepam) into the active site of $\mathrm{GABA}_{\mathrm{A}}$ receptor enzyme reveals that it occupies the same binding pocket with RMSD of $0.41 \mathrm{~A}$, which further validates the present docking protocol. Docking results revealed that the highest docking score bound tightly in the active site of $\mathrm{GABA}_{\mathrm{A}}$ receptor and makes a hydrogen bond with the backbone of amino acid residue vizAla201 and Thr202. Furthermore, on the docking analysis of the highest docking score compound with standard ligand (diazepam) by superimposition, it gave the same interaction pattern of binding with the catalytic domain of GABA receptor enzyme. The highest docking score was observed to be -3.22 ) than the diazepam $(-3.21)$ against $\mathrm{GABA}_{\mathrm{A}}$ receptor.

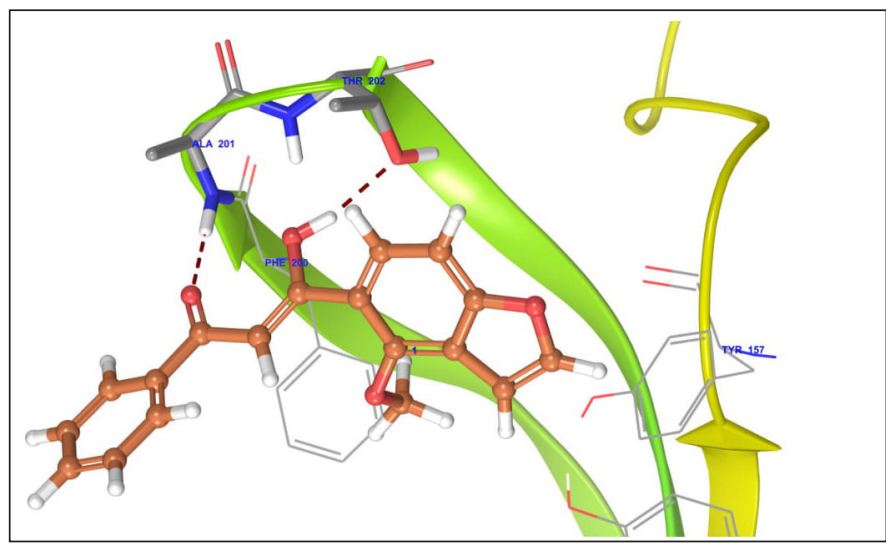

Figure 2. Binding interaction of highest docking score against $\mathrm{GABA}_{\mathrm{A}}$ receptor (Pdbid-4COF)

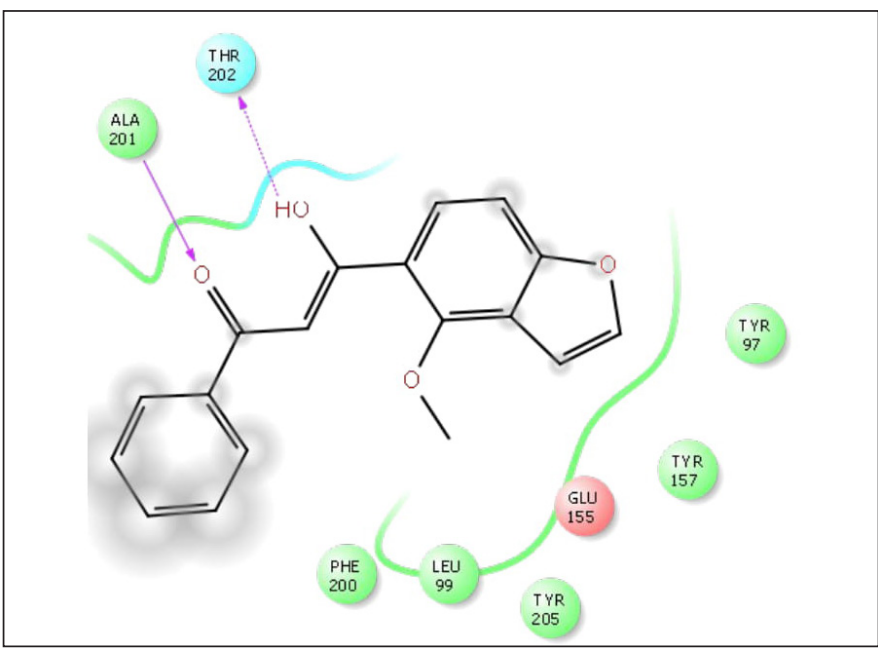

Figure 3. Ligplot of highest docking score against $\mathrm{GABA}_{\mathrm{A}}$ Receptor (Pdbid4COF).

Table 1. Effect of MPD on the locomotor activity of mice using actophotometer.

\begin{tabular}{lcccc}
\hline \multirow{2}{*}{ Treatment } & Dose (mg/kg) & \multicolumn{3}{c}{ Locomotor activity after treatment (minute) } \\
\cline { 3 - 5 } & & $\mathbf{3 0}$ & $\mathbf{6 0}$ & $\mathbf{9 0}$ \\
\hline Control & DMSO 1\% & $65.36 \pm 0.58$ & $62.66 \pm 0.66$ & $58.45 \pm 0.62$ \\
Diazepum & 2 & $14.18 \pm 0.52$ & $22.5 \pm 0.47^{*}$ & $19.98 \pm 0.35^{*}$ \\
Drug & 25 & $64 \pm 0.66$ & $51.66 \pm 0.33$ & $50.89 \pm 1.24$ \\
& 50 & $49.35 \pm 0.56$ & $36.38 \pm 0.56^{*}$ & $34.66 \pm 1.15^{*}$ \\
& 100 & $31.44 \pm 0.41$ & $23.66 \pm 0.79^{*}$ & $20.45 \pm 0.67^{*}$ \\
\hline
\end{tabular}

Results are expressed as mean \pm S.E.M $(n=6)$. The values were compared with the control group. Significant variation against control $* p<0.01$ (analysis of variance test followed one-way analysis of variance (ANOVA) followed by Dunnett's ' $t$ ' test).

Table 2. Anxiolytic effect of MPD using elevated plus maze apparatus on mice.

\begin{tabular}{lccccccc}
\hline \multirow{2}{*}{ Treatment } & \multirow{2}{*}{ Dose (mg/kg) } & \multicolumn{3}{c}{ Time spent in closed arm } & \multicolumn{3}{c}{ No. of entries to open arm } \\
\cline { 3 - 7 } & & $\mathbf{3 0}$ & $\mathbf{6 0}$ & $\mathbf{1 2 0}$ & $\mathbf{3 0}$ & $\mathbf{6 0}$ & $\mathbf{1 2 0}$ \\
\hline Control & DMSO 1\% & $190.74 \pm 3.14$ & $187.46 \pm 1.29$ & $183.37 \pm 1.69$ & $1.83 \pm 0.26$ & $2.33 \pm 0.27$ & $2.66 \pm 0.56$ \\
Diazepam & 2 & $147.25 \pm 1.26$ & $145.67 \pm 2.45$ & $135.44 \pm 3.13$ & $10.5 \pm 1.26^{*}$ & $12.66 \pm 1.89^{* *}$ & $14.3 \pm 3.47^{* *}$ \\
Drug & 25 & $157.63 \pm 1.67$ & $149.89 \pm 2.46$ & $140.37 \pm 2.49$ & $8.5 \pm 1.86$ & $9.5 \pm 1.73^{*}$ & $9.13 \pm 1.49^{*}$ \\
& 50 & $152.36 \pm 3.26$ & $148.54 \pm 1.29$ & $138.35 \pm 3.16$ & $8.75 \pm 1.63$ & $10.76 \pm 1.45^{*}$ & $10.54 \pm 1.77^{*}$ \\
& 100 & $149.34 \pm 1.89$ & $146.25 \pm 2.85$ & $136.67 \pm 3.19$ & $9.25 \pm 1.89^{*}$ & $11.33 \pm 1.67^{* *}$ & $12.66 \pm 1.49^{* *}$ \\
\hline
\end{tabular}

Results are expressed as mean \pm S.E.M $(n=6)$. The values were compared with the control group. Significant variation against control and Significant variation against control $* p<0.01$ (Analysis of variance test followed one-way ANOVA by Dunnett's ' $t$ ' test). 


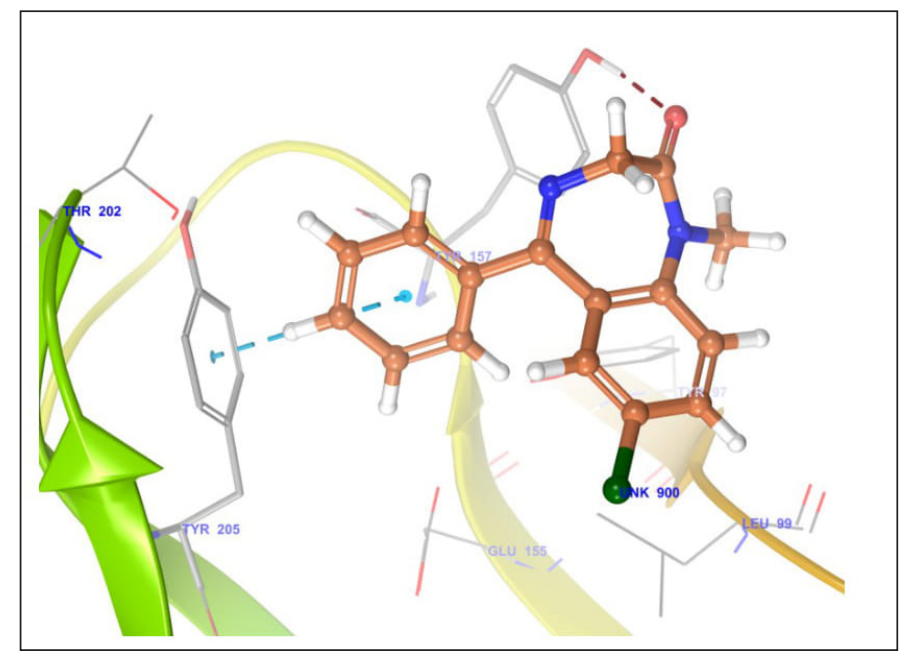

Figure 4. Binding interaction of standard diazepamagainst $\mathrm{GABA}_{\mathrm{A}}$ (Pdbid4COF).

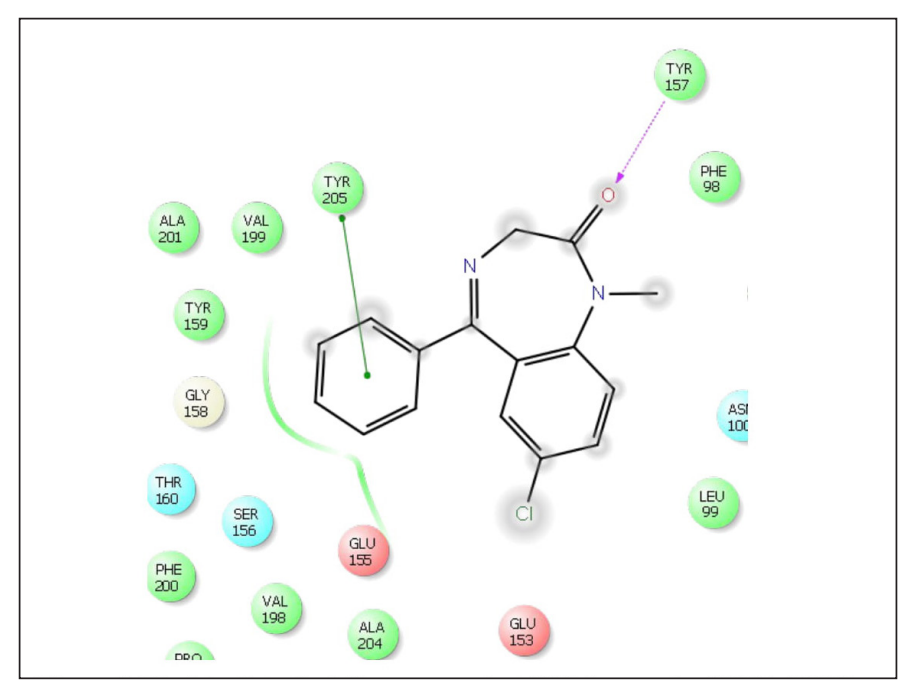

Figure 5. Ligplot of standard diazepam against GABAA receptor (Pdbid-4COF).

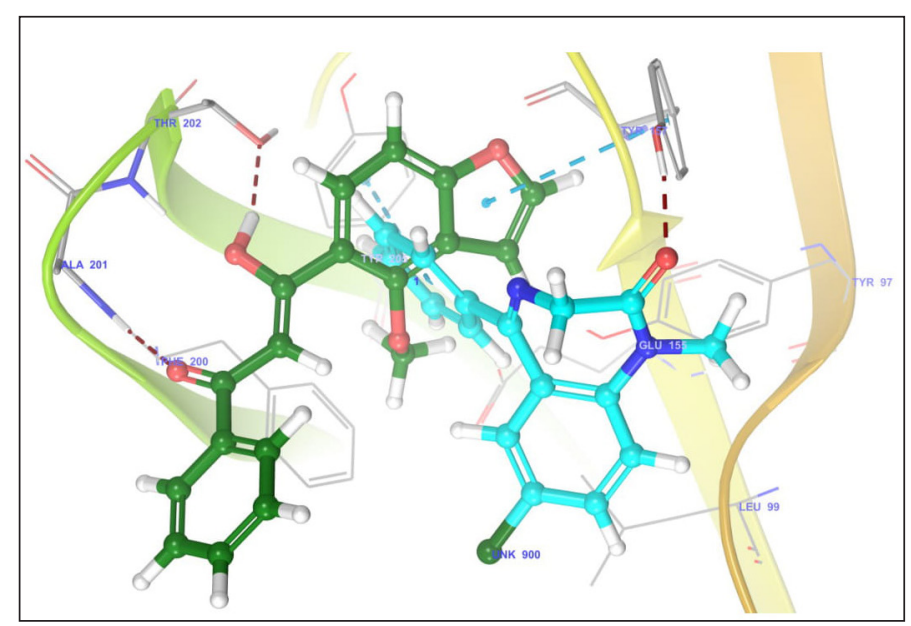

Figure 6. Superimposition of compound highest docking score (Green) with standard diazepam (Cyan) against GABAA receptor (PDBID-4COF).

\section{CONCLUSION}

The CNS-depressant effect of MPD from gross behavior study and the molecular structure initiated the anxiolytic activity evaluation of MPD. The study of compound MPD has observed to have significant anxiolytic activity from an animal study. MPD showed a significantly reduced locomotion which reflects its depressant effect on CNS. Further from its observation, the use of elevated plus confirmed its anxiolytic activity which was observed to be dose dependent. The pharmacological study is well corroborated with docking studies with an understanding of the interactions in the active site of $\mathrm{GABA}_{\mathrm{A}}$ receptor. The docking results revealed that the MPD bound tightly in the active site of $\mathrm{GABA}_{\mathrm{A}}$ receptor. This compound MPD well occupied in the receptor cavity and forms hydrogen bonds and hydrophobic interactions. The mechanism behind the anxiolytic effect of MPD is confirmed to be due to the binding and stimulation of $\mathrm{GABA}_{\mathrm{A}}$ receptor, and it reduced the anxiety symptoms. The study concluded that the potent anxiolytic effect of MPD is by binding to $\mathrm{GABA}_{\mathrm{A}}$ receptor which is well comparable to that of the standard drug diazepam. Furthermore, formulation study and clinical study will be helpful to make available for the treatment of anxiety. MPD will really be helpful to mitigate the untoward health issue such as anxiety.

\section{ACKNOWLEDGMENTS}

The authors are acknowledging to Dr. Dinakar Sasmal for his constant motivation toward the research. The Managing Director, Additional Managing Director, and Executive President, Noida Institute of Engineering and Technology are also acknowledged for providing the research facility.

\section{CONFLICT OF INTEREST}

Authors declared that there is no conflicts of interest.

\section{FUNDING}

None.

\section{REFERENCES}

Anderson AC. The process of structure-based drug design. Chem Bio, 2003; 10:787-89.

Basu SP, Mandal JK, Mehdi NS. A review of pharmacology of phytochemicals from Indian medicinal plants. Ind J Pharm Sci, 1994, $56: 163$.

Bhatia G, Puri A, Maurya R, Yadav PP, Khan MM, Khanna AK. Antidislipidemic and antioxidant activities of different fractions of Pongamia pinnata. Med Chem Res. 2008; 17:281-87.

Friesner RA, Murphy RB, Repasky MP, Frye LL, Greenwood JR, Halgren TA, Sanschagrin PC, Mainz DT. Extra precision glide: docking and scoring incorporating a model of hydrophobic enclosure for proteinligand complexes. J Med Chem, 2006; 49:6177-96.

Ghosh MN. Fundamentals of experimental pharmacology. 2nd edition, Scientific Book Agency, Kolkata, India, pp 153-58, 1984.

Jones G, Willett P, Glen RC, Leach AR, Taylor R. Development and validation of a genetic algorithm for flexible docking. J Mol Bio, 1997; 267:727.

Katsila T, Spyroulias GA, Patrinos GP, Matsoukas MT. Computational approaches in target identification and drug discovery. Comput Str Biotech J, 2016; 14:177-82.

Kirtikar KR, Basu BD. Indian Medicinal Plants. International book distributors, Dehardun, India, 1: pp 830-832, 1995.

Kumar AT, Jaiswal N, Yadav PP, Maurya R, Srivastava AK Pongamol from pongamia pinnata stimulates glucose uptake by increasing surface GLUT4 level in skeletal muscle cells. Mol Cell Endocrinol, 2011; 339(1-2):98-104. 
Kumar PA, Das S, Mohapatra S, Basu SP, Sharma K.Antipsychotic potential and molecular modelling of 1-(4-methoxybenzofuran-5-yl)-3phenylpropane-1, 3-dione. J Gujarat Res Soc, 2019; 21(13):362-70.

Kumar PA, Mohapatra S, Das S, Basu SP. Novel synthetic and commercial oriented synthesis of substituted benzofuran moiety. J Innov Pharm Biol Sci, 2016; 3:116-19.

Lydiard RB. The role of GABA in anxiety disorders. J Clin Psychiatry, 2003; 64(3):21-7.

Mohammad A. Mini review on important biological properties of benzofuran derivatives. J Anal Pharm Res, 2016; 3(2):50-6.

Nuss P, Gauthier I. Anxiety disorders and GABA neurotransmission: a disturbance of modulation. Neuropsychiatr Dis Treat, $2015 ; 11: 165-75$.

Ramdhave AS, Badole SL, Bodhankar SL. Anticonvulsant activity of stem bark of Pongamia pinnata. Biomed Aging Pathol, 2011; 1(3):147-57.

Rao RR, Chaturvedi V, Babu SK, Reddy PP, SubbaRao VR, Sreekanth P, Sreedhar AS, Rao JM. Synthesis and anticancer effects of pongamol derivatives on mitogen signaling and cell cycle kinases. Med Chem Res. 2001; 21(5):634-39.
Turner RA. Screening methods of pharmacology. Acd Press Inc, New York, NY and London, UK, p 32, 1965.

Viswanadhan VN, Ghose AK, Revankar GK, Robins RK. Atomic physicochemical parameters for three dimensional structure directed quantitative structure-activity relationships. Additional parameters for hydrophobic and dispersive interactions and their application for an automated superposition of certain naturally occurring nucleoside antibiotics. J Chem Inf Comput Sci, 1989; 29:163-72.

How to cite this article:

Kumar PA, Das S, Mohapatra S, Basu SP, Sharma K, Sharma M, Singh AP. Molecular modeling, synthesis, and evaluation of 1-(4-methoxybenzofuran-5-yl)-3-phenylpropane-1, 3-dione for its anxiolytic potentiality. J Appl Pharm Sci, 2020; 10(08):139-144. 